\title{
Correspondence
}

Editor: Ian Pullen

Contents: Sibling sex and bulimia nervosa/Strength of association/The future of psychotherapy/Child psychiatry in the 20th century (England \& Wales)/ Evaluation of motor disorder in mentally handicapped people/Safety of 5-HT reuptake inhibitors/ Post-partum psychoses and breast feeding in developing countries/Spousal allegations of incest during transient psychotic episodes/Fitness to plead/ Obsessive slowness revisited/Musical hallucinations triggered by clomipramine/Crossover reaction between haloperidol and amoxapine for NMS.

\section{Sibling sex and bulimia nervosa}

SIR: We had written previously about the paper by Lacey et al (Journal, April 1991, 158, 491-494) but withdrew our letter given the considerable overlap with the points made by Goodman (Journal, August 1991, 159, 290). However, we feel we must write again given the serious deficiencies of the authors' reply to Dr Goodman's criticisms (Journal, August $1991,159,291)$, It seems that Dr Lacey and colleagues are attempting to obfuscate a rather simple issue with a mathematical formula and that they remain unaware of the basic error they have made.

To return to the original illustration in their paper, they state that, making no assumption about the position of the female in the sibship, the possibilities for a three-sibship family with at least one girl are as follows: MMF, MFM, FMM, FMF, FFM and FFF. In this case, then, a female who has two brothers is counted as having three different potential family constellations (MMF, MFM and FMM), while a girl who has two sisters is counted as having only one possible family constellation (FFF). Thus, position in sibship is not taken into account when considering all female sibships but it is taken into account when considering other types of sibship constellation.

Dr Lacey and colleagues calculate (given that one sibling is a girl) the odds against all-girl sibship sizes of $2,3,4$ and 5 respectively to be one in 2 , one in 7 , one in 15 and one in 31 , whereas these should read one in 2 , one in 4 , one in 8 and one in 16. The main conclusions of the paper are based on these faulty statistics, and after the appropriate corrections are made, the excess of girls appears to be much more modest and probably not significantly different from normal sex distribution within sibships. Indeed, in making such calculations, it would be optimal to bear in mind that, with successive births of siblings of the same sex, the odds continue to increase in favour of the next sibling being of the same sex (James, 1987).

JAmes, W. H. (1987) The human sex ratio Part I: A review of the literature. Human Biology, 59, 721-752.

JoHN M. EAGLES

Ross Clinic MAUREEN I. JOHNSTON

Cornhill Road

Aberdeen $A B 92 Z F$

SIR: I expect you will receive a lot of correspondence on the confusion of elementary statistics evident in the letter and reply "Sibling sex and bulimia nervosa" (Journal, August 1991, 159, 290-291). Evidently, one or other eminent authority is in error in their reasoning. I would incline to the reasoning of Goodman, since that of Lacey et al seems to assume that onethird of their initially female subjects may in fact have been males. The ease with which apparently logical statistical arguments can lead one horribly astray is one reason I abandoned university level mathematics in my youth. It would seem that even at this very basic level we psychiatrists are in need of a statistician's expert opinion.

\section{G. N. CONACHER}

Regional Treatment Centre

Kingston Penitentiary

P.O. Box 22

Kingston, Ontario

K7L $4 V 7$

\section{Strength of association}

SIR: Since any single piece of research is necessarily flawed, correspondence about a study can become excessive. However, Thomas's (Journal, August 1991, 159, 292-293) recent elaboration of a point made by Muijen (Journal, May 1991, 158, 713) requires comment. The subject study by Johnstone et al (Journal, August 1990, 157, 182-189) reported that 